\title{
Langmuir
}

pubs.acs.org/Langmuir

(C) 2009 American Chemical Society

\section{Fabrication of Artificial Opals by Electric-Field-Assisted Vertical Deposition}

\author{
Kirill S. Napolskii, ${ }^{, \dagger}$ Nina A. Sapoletova,${ }^{\dagger}$ Dmitriy F. Gorozhankin, ${ }^{\dagger}$ Andrey A. Eliseev, ${ }^{\dagger}$ \\ Dmitry Yu. Chernyshov, ${ }^{\star}$ Dmytro V. Byelov, ${ }^{\S}$ Natalia A. Grigoryeva,", Alexander A. Mistonov," \\ Wim G. Bouwman, ${ }^{\perp}$ Kristina O. Kvashnina, ${ }^{\#}$ Alexey V. Lukashin, ${ }^{\dagger}$ Anatoly A. Snigirev, ${ }^{\#}$ \\ Alexandra V. Vassilieva, ${ }^{\nabla}$ Sergey V. Grigoriev, ${ }^{\nabla}$ and Andrei V. Petukhov ${ }^{\S}$ \\ ${ }^{\dagger}$ Department of Materials Science, Moscow State University, Leninskie Hills, 119991 Moscow, Russia, \\ ${ }^{*}$ Swiss-Norwegian Beamlines at the European Synchrotron Radiation Facility, BP220, F-38043 Grenoble Cedex, \\ France, ${ }^{\S}$ Van't Hoff Laboratory for Physical and Colloid Chemistry, Debye Institute for Nanomaterials Science, \\ University of Utrecht, Padualaan 8, 3584 CH Utrecht, The Netherlands, "Department of Physics, St. Petersburg \\ State University, 198504 St. Petersburg, Russia, ${ }^{\perp}$ Faculty of Applied Sciences, Delft University of Technology, \\ Mekelweg 15, 2629 JB Delft, The Netherlands, \# European Synchrotron Radiation Facility, BP220, F-38043 \\ Grenoble Cedex, France, and ${ }^{\nabla}$ Petersburg Nuclear Physics Institute, Gatchina, 188300 St. Petersburg, Russia
}

Received July 29, 2009. Revised Manuscript Received September 20, 2009

\begin{abstract}
We present a new technique for large-scale fabrication of colloidal crystals with controllable quality and thickness. The method is based on vertical deposition in the presence of a DC electric field normal to the conducting substrate. The crystal structure and quality are quantitatively characterized by microradian X-ray diffraction, scanning electron microscopy, and optical reflectometry. Attraction between the charged colloidal spheres and the substrate promotes growth of thicker crystalline films, while the best-quality crystals are formed in the presence of repulsion. Highly ordered thick crystalline layers with a small amount of stacking faults and a low mosaic spread can be obtained by optimizing the growth conditions.
\end{abstract}

\section{Introduction}

Photonic crystals are materials with a modulated refractive index on the scale of the order of light wavelengths in the visible or near-infrared region of the spectrum. These materials attract great attention due to optical properties that allow one to control and manipulate the flow of light. ${ }^{1,2}$ Despite the significant progress achieved in understanding the optical phenomena in photonic crystals, large-scale fabrication of high-quality photonic crystals remains a challenge.

The use of colloidal crystals for the production of $3 \mathrm{D}$ photonic crystals is a fast and cheap pathway that gives large-scale periodic structures. Colloidal self-assembly, however, often yields a high density of defects, which can significantly affect the optical properties by breaking the average symmetry of the crystal and creating defect states. ${ }^{2-4}$ The quality of colloidal crystals leaves much to be desired in comparison with structures obtained by lithography ${ }^{5,6}$ or holography ${ }^{7,8}$ techniques.

*Corresponding author. E-mail: napolsky@inorg.chem.msu.ru.

(1) Yablonovitch, E. Phys. Rev. Lett. 1987, 58, 2059.

(2) John, S. Phys. Rev. Lett. 1987, 58, 2486

(3) Vlasov, Yu. A.; Astratov, V. N.; Baryshev, A. V.; Kaplyanskii, A. A.; Karimov, O. Z.; Limonov, M. F. Phys. Rev. E 2000, 61, 5784-5793.

(4) Rengarajan, R.; Mittleman, D.; Rich, C.; Colvin, V. Phys. Rev. E 2005, 71, 016615 .

(5) Lin, S. Y.; Fleming, J. G.; Hetherington, D. L.; Smith, B. K.; Biswas, R.; Ho, K. M.; Sigalas, M. M.; Zubrzycki, W.; Kurtz, S. R.; Bur, J. Nature 1998, 394, 251253.

(6) Noda, S.; Tomoda, K.; Yamamoto, N.; Chutinan, A. Science 2000, 289, 604606.

(7) Sun, H. B.; Matsuo, S.; Misawa, H. Appl. Phys. Lett. 1999, 74, 786-788.

(8) Campbell, M.; Sharp, D. N.; Harrison, M. T.; Denning, R. G.; Turberfield,

A. J. Nature 2000, 404, 53-56.

(9) Waterhouse, G. I. N.; Waterland, M. R. Polyhedron 2007, 26, 356368 .

(10) Miguez, H.; Lopez, C.; Meseguer, F.; Blanco, A.; Vazquez, L.; Mayoral, R.; Ocana, M.; Fornes, V.; Mifsud, A. Appl. Phys. Lett. 1997, 71, 1148-1150.
Nowadays, various methods to fabricate colloidal crystals have been developed such as sedimentation, ${ }^{9,10}$ spin coating, ${ }^{11}$ colloidal epitaxy, ${ }^{12,13}$ electrophoresis, ${ }^{14-16}$ and convective assembly, also known as controlled drying or vertical deposition. ${ }^{17-21}$ All these techniques yield large arrays of colloidal particles, but cracks, stacking faults, dislocations, and point defects are still abundant. Moreover, reproducibility of the results is often a problem.

In the case of vertical deposition, a number of competing forces are involved in the colloid self-assembly. Capillary forces are very strong but act only on the particles at the liquid-air interface. In the liquid bulk, it is the combination of the convection, sedimentation, diffusion, and interparticle forces that determine the particle trajectories. Recently, the relation between the growth dynamics and the structural quality of colloidal crystal films has been established using optical reflectometry. ${ }^{22,23}$ Photonic crystal

(11) Jiang, P.; McFarland, M. J. J. Am. Chem. Soc. 2004, 126, 13778-13786.

(12) Hoogenboom, J. P.; van Langen-Suurling, A. K.; Romijn, J.; van Blaaderen, A. Phys. Rev. E 2004, 69, 051602.

(13) van Blaaderen, A.; Ruel, R.; Wiltzius, P. Nature 1997, 385, 321-324.

(14) Trau, M.; Saville, D. A.; Aksay, I. A. Langmuir 1997, 13, 6375-6381.

(15) Rogach, A. L.; Kotov, N. A.; Koktysh, D. S.; Ostrander, J. W.; Ragoisha,

G. A. Chem. Mater. 2000, 12, 2721-2726.

(16) Holgado, M.; Garcia-Santamaria, F.; Blanco, A.; Ibisate, M.; Cintas, A.; Miguez, H.; Serna, C. J.; Molpeceres, C.; Requena, J.; Mifsud, A.; Meseguer, F.; Lopez, C. Langmuir 1999, 15, 4701-4704.

(17) Jiang, P.; Bertone, J. F.; Hwang, K. S.; Colvin, V. L. Chem. Mater. 1999, 11, 2132-2140.

(18) Blanco, A.; Chomski, E.; Grabtchak, S.; Ibisate, M.; John, S.; Leonard, S. W.; Lopez, C.; Meseguer, F.; Miguez, H.; Mondia, J. P.; Ozin, G. A.; Toader, O.; van Driel, H. M. Nature 2000, 405, 437-440.

(19) Norris, D. J.; Arlinghaus, E. G.; Meng, L.; Heiny, R.; Scriven, L. E. Adv. Mater. 2004, 16, 1393-1399.

(20) Meng, L.; Wei, H.; Nagel, A.; Wiley, B. J.; Scriven, L. E.; Norris, D. J. Nano Lett. 2006, 6, 2249-2253.

(21) Wei, H.; Meng, L.; Jun, Y.; Norris, D. J. Appl. Phys. Lett. 2006, 89, 241913.

(22) Lozano, G.; Miguez, H. Appl. Phys. Lett. 2008, 92, 091904.

(23) Lozano, G. S.; Dorado, L. A.; Depine, R. A.; Miguez, H. J. Mater. Chem. 2009, 19, 185-190. 
engineering can use a number of parameters, which can influence the forces responsible for the formation of a crystal. For example, by adjusting the density mismatch between the particles and the solvent, one can modify the strength of the effective gravity force. Variation of the growth temperature can slightly affect the diffusion and can have a strong effect on the evaporation rate and, therefore, on the convection force. Changing the particle charge can influence the interparticle forces. Still, one may need to have additional means to vary the force balance and, therefore, to fine tune the crystal structure and quality.

The electric field could be an excellent tool to control the crystallization process. It was shown that the electric field can act as the main driving force, which moves colloidal particles to the substrate and induces the crystallization process. ${ }^{14,15}$ The electric field can also play an auxiliary role by, for example, activating shear deformations and therefore improving the crystal structure during the formation of artificial opals by the inclined deposition method. ${ }^{24}$ The electric field was successfully utilized for preparation of colloidal crystals from monodisperse ${ }^{14}$ and even binary colloidal particles. ${ }^{25}$ It was also used to induce dipole-dipole interparticle interactions, which can improve the crystal quality and even change the crystal structure. ${ }^{26}$

Here we present results on the application of the DC electric field normal to the substrates during vertical deposition of colloidal crystals. To the best of our knowledge, for such a geometry, the influence of the electric field on the structure of colloidal crystals has not been studied before. Moreover, investigations of the formation of colloidal crystals in an electric field so far have been performed for rather thin structures. ${ }^{14,24}$ The stacking order is mostly addressed using electron ${ }^{15}$ or optical ${ }^{27}$ microscopy, which are local and can be applied to very limited sample volumes. Here we show that a detailed quantitative characterization of structural order as a function of the electric field can be performed by combining scanning electron microscopy, high resolution microradian X-ray diffraction, and optical reflectometry. The single crystal X-ray diffraction on colloidal crystals was first reported in ref 28 . Since then, this method has been successfully used for the characterization of the structural order of artificial opals. ${ }^{29-31}$ Despite the negative charge of the particles, the crystals are found to form on both positively and negatively charged electrodes. It is found that electrostatic attraction between the particles and the substrate increases the crystal thickness while repulsion between them promotes higher crystal quality. Optical reflectometry also is used to illustrate the effect of the crystal quality on its optical properties.

\section{Experimental Section}

Materials and Substrates. Styrene $\left(\mathrm{C}_{6} \mathrm{H}_{5} \mathrm{CH}=\mathrm{CH}_{2}, 99.5 \%\right)$ and potassium persulfate $\left(\mathrm{K}_{2} \mathrm{~S}_{2} \mathrm{O}_{8}, 99.99 \%\right)$ were obtained from Sigma-Aldrich. Pure water $(>18 \mathrm{M} \Omega \cdot \mathrm{cm})$ was used directly from a Milli-Q water system. Glass slides covered with an indium tin oxide (ITO) layer with a surface resistivity of $15-25 \Omega /$ sq were

(24) Schope, H. J. J. Phys.: Condens. Matter 2003, 15, L533-L540.

(25) Ristenpart, W. D.; Aksay, I. A.; Saville, D. A. Phys. Rev. Lett. 2003, 90, 128303 .

(26) Yethiraj, A.; Wouterse, A.; Groh, B.; van Blaaderen, A. Phys. Rev. Lett. 2004, 92,058301

(27) Zhang, K. Q.; Liu, X. Y. J. Chem. Phys. 2009, 130, 184901.

(28) Vos, W. L.; Megens, M.; van Kats, C. M.; Bösecke, P. Langmuir 1997, 13, 6004-6008.

(29) Petukhov, A. V.; Aarts, D. G.; Dolbnya, I. P.; De Hoog, E. H.; Kassapidou,

K.; Vroege, G. J · Bras, W ; Lekkerkerker, H. N. Phys. Rev. Lett. 2002, 88, 208301.

(30) Thijssen, A. V.; Petukhov, A. V.; 't Hart, D. C.; Imhof, A.; van der Werf,

C. H. M.; Schropp, R. E. I.; van Blaaderen, A. Adv. Mater. 2006, 18, 1662-1666.

(31) Abramova, V. V.; Sinitskii, A. S.; Grigor'eva, N. A.; Grigor'ev, S. V.; Belov, D. V.; Petukhov, A. V.; Mistonov, A. A.; Vasil'eva, A. V.; Tret'yakov, Y. D. J. Exp. Theor. Phys. 2009, 109, 29-34. purchased from $3 \mathrm{M}$. The slides were cut into $4 \mathrm{~cm} \times 1.5 \mathrm{~cm}$ pieces and used as substrates for deposition of artificial opals.

Sample Preparation. The polystyrene (PS) microspheres were synthesized by emulsifier-free emulsion polymerization of styrene using potassium persulfate as an initiator. ${ }^{32}$ Prior to the polymerization, styrene was purified from a stabilizer (4-tertbutylcatechol) by vacuum distillation. The mixture with molar ratio $1 \mathrm{C}_{8} \mathrm{H}_{8} / 0.003 \mathrm{~K}_{2} \mathrm{~S}_{2} \mathrm{O}_{8} / 58 \mathrm{H}_{2} \mathrm{O}$ was vigorously stirred for $24 \mathrm{~h}$ at $343 \mathrm{~K}$.

Colloidal crystals made of polystyrene microspheres were grown by the vertical deposition technique in the presence of an external electric field. Deposition of negatively charged PS spheres onto ITO glass vertically aligned electrodes was performed in a cylindrical glass cell $(5 \mathrm{~cm}$ diameter $)$ using a Solartron 1287 potentiostat. The distance between the electrodes was $3 \mathrm{~cm}$. The electrodes were fixed precisely parallel to each other. Before the deposition, ITO glass substrates were carefully washed under sonication in pure grade ethanol and water. The concentration of PS particles in the colloidal solution was $\sim 0.2 \mathrm{vol} \%$, and the $\mathrm{pH}$ of the mixture was 4. Deposition of PS spheres has been carried out at constant voltage $U$ ranging from 0.1 to $3 \mathrm{~V}$ for $24 \mathrm{~h}$. The temperature of the film growth was $60 \pm 3{ }^{\circ} \mathrm{C}$.

Sample Characterization. Scanning electron microscopy (SEM) pictures were recorded on a Supra $50 \mathrm{VP}$ instrument (LEO). Samples for SEM were covered by a thin conductive layer of carbon using a Scancoat sputterer (Edwards). A Lambda 950 spectrophotometer (Perkin-Elmer) is used to record optical reflection spectra at incident angle of $8^{\circ}$ with respect to the normal to the sample. The size of the light spot was $4 \times 4 \mathrm{~mm}^{2}$.

$\mathrm{X}$-ray studies were performed at the Dutch-Belgian beamline BM-26 DUBBLE of the European synchrotron radiation facility (ESRF) in Grenoble (France) using a microradian X-ray diffraction setup similar to the one described in ref 33 . In brief, to achieve the maximum transverse coherence length of the beam, any focusing of the beam before the experimental hutch was avoided. Instead, the X-ray beam was focused by a set of compound refractive lenses (CRLs) ${ }^{34}$ at the phosphor screen of the CCD (charge-coupled device) X-ray detector (Photonic Science VHR, $4008 \times 2671$ pixels of $9 \times 9 \mu \mathrm{m}^{2}$ ) located at a distance of $8 \mathrm{~m}$ from the lenses. The samples were placed just after the CRLs. This scheme, which differs from an ordinary small-angle X-ray scattering setup by the novel approach to beam focusing, allows one to achieve angular resolution (full-width at half-maximum) of the order of $5 \mu \mathrm{rad}$, corresponding to $3.3 \times 10^{-4} \mathrm{~nm}^{-1}$ in the reciprocal space. A $12 \mathrm{keV} X$-ray beam (wavelength $\lambda=0.1 \mathrm{~nm}$, band-pass $\Delta \lambda / \lambda=2 \times 10^{-4}$, size $0.5 \times 0.5 \mathrm{~mm}^{2}$ at the sample position) was used. The colloidal films were first mounted perpendicular to the X-ray beam. Samples were then rotated around the vertical axis within the range $-75^{\circ} \leq \omega \leq 75^{\circ}$, and the diffraction patterns were recorded at each degree of rotation. The collected images represent sections of reciprocal space projected on the flat area detector. This allowed for the mapping of most of the three-dimensional reciprocal lattice of the crystal. It is worth noting that the curvature of Ewald's sphere in smallangle experiments could be neglected due to the gigantic difference between the structure period and the X-ray wavelength. The background subtraction and reconstruction of reciprocal space from the scattering data were performed by a Mathcad code developed in-house. ${ }^{35}$

(32) Goodwin, J. W.; Hearn, J.; Ho, C. C.; Ottewill, R. H. Colloid Polym. Sci. 1974, 252, 464-471.

(33) Petukhov, A. V.; Thijssen, J. H. J.; ’t Hart, D. C.; Imhof, A.; van Blaaderen, A.; Dolbnya, I. P.; Snigirev, A.; Moussaid, A.; Snigireva, I. J. Appl. Crystallogr. 2006, 39, 137-144.

(34) Snigirev, A.; Kohn, V.; Snigireva, I.; Lengeler, B. Nature 1996, 384, 49-51. (35) Eliseev, A. A.; Gorozhnkin, D. F.; Napolskii, K. S.; Petukhov, A. V.; Sapoletova, N. A.; Vassilieva, A. V.; Grigoryeva, N. A.; Mistonov, A. A.; Beylov, D. V.; Bouwman, W. G.; Kvashnina, K. O.; Chernyshov, D. Yu.; Bossak, A. A.; Grigoriev, S. V. JETP Lett. 2009, 90, 297-303. 

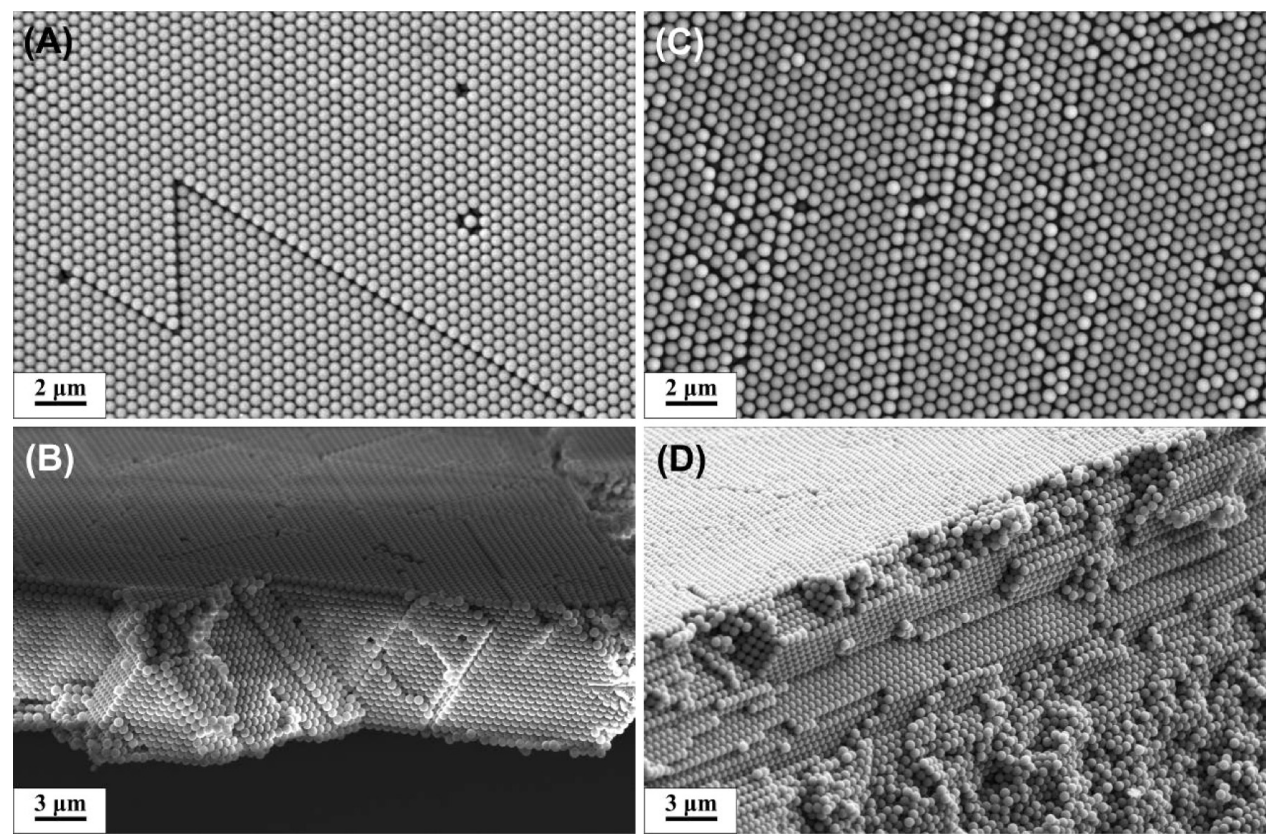

Figure 1. SEM images of colloidal crystals prepared by vertical deposition method in the presence of an external electric field perpendicular to the substrates. Panels A and B correspond to the top surface and cross section of the crystal deposited at $U=1.5 \mathrm{~V}$ on a cathode, respectively, and $\mathrm{C}$ and $\mathrm{D}$ at $U=3 \mathrm{~V}$ on an anode. The distance between electrodes is $3 \mathrm{~cm}$.

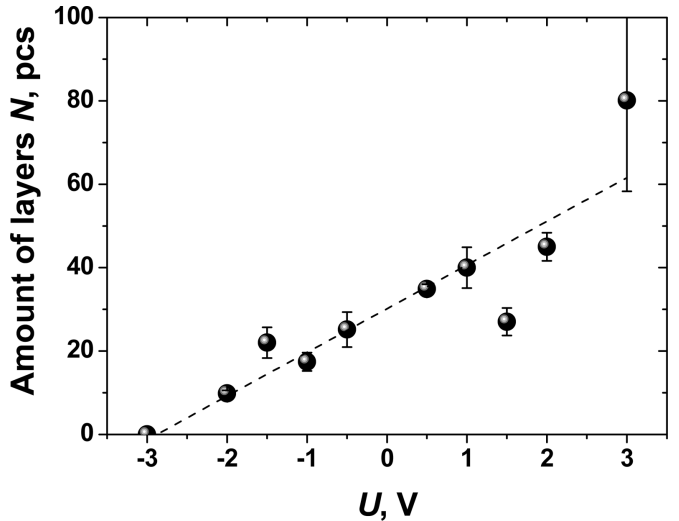

Figure 2. Dependence of the thickness of a colloidal film on applied voltage $U$ (according to SEM data). Negative values of $U$ correspond to a cathode polarization, and positive to an anode polarization. Error bars indicate a standard deviation from the mean value. The dashed line is the linear fit to the experimental data.

\section{Results and Discussion}

The representative scanning electron micrographs of as-grown colloidal crystals of polystyrene spheres obtained on a cathode and on an anode are shown in Figure 1. Panels A and B correspond to the top surface and cross section of the crystal deposited at $U=1.5 \mathrm{~V}$ on a cathode, respectively, and $\mathrm{C}$ and $\mathrm{D}$ at 3 $\mathrm{V}$ on an anode. On the cathode, one can see that the particles are neatly arranged into periodic arrays. Still, some defects are visible such as a few vacancies and lines (Figure 1A), where the spheres have a squarelike arrangement. The apparent line defects are typical for crystals grown by convective assembly ${ }^{4,36}$ and are openings of double stacking faults at the angle of $70.5^{\circ}$ with the substrate. ${ }^{36}$

(36) Hilhorst, J.; Abramova, V. V.; Sinitskii, A.; Sapoletova, N. A.; Napolskii, K. S.; Eliseev, A. A.; Byelov, D. V.; Grigoryeva, N. A.; Vasilieva, A. V.; Bouwman, W. G.; Kvashnina, K.; Snigirev, A.; Grigoriev, S. V.; Petukhov, A. V. Langmuir 2009, 25, 10408-10412.
Figure 1C illustrates that anode polarization leads to formation of highly defective colloidal crystal structure. In this case, the electrostatic attraction between negatively charged particles and the positive electrode is presumably too strong, so that the polystyrene spheres have little chance to rearrange their position to find a better place in the crystal. Only top layers of the formed films are ordered due, probably, to screening of Coulomb interaction by the bottom layers (Figure 1D). Sample cross sections seen in SEM reveal that the thickness of colloidal films formed on the cathode $(-)$ is smaller than the corresponding value for crystals on the anode $(+)$. The dependence of film's thickness on applied voltage is shown in Figure 2.

To quantify the effect of the electric field on the structural order of colloidal films, we complemented the SEM observations with microradian X-ray diffraction accompanied by 3D mapping of the reciprocal space. ${ }^{35}$ Figure 3 shows typical examples of microradian diffraction patterns measured for different orientations of colloidal crystals, corresponding to lowest index zones of fcc structure, that is, (111) at $\omega=0^{\circ},(101)$ at $\omega=-35.3^{\circ}$, and (010) at $\omega=54.7^{\circ}(\omega$ is the rotation angle around the vertical axis, which aligns with the [101] axis of the crystal). In the diffraction patterns, one can clearly identify a large number of Bragg reflections, which can be assigned to the reciprocal lattice of the ideal fcc crystal structure with the cubic cell size of $a_{0}=750 \mathrm{~nm}$. Corresponding indexes are shown in Figure 3.

In addition to the assigned Bragg peaks, the diffraction patterns also show features which cannot be assigned to the fcc structure (marked by arrows in Figure 3A). They can be truly Bragg's reflexes (e.g., corresponding to an hep structure coexisting with fcc), or can be sections of diffuse objects in the reciprocal space. The latter can be related to the finite film thickness and/or to the presence of stacking faults along $\langle 111\rangle$ cubic directions. ${ }^{37}$ Complete information on this type of disorder could be inferred from the distribution of diffracted intensity in the three-dimensional (3D) reciprocal space. The latter is reconstructed from the collected diffraction data. ${ }^{35}$ An example of a $3 \mathrm{D}$ map in reciprocal

(37) Loose, W.; Ackerson, B. J. J. Chem. Phys. 1994, 101, 7211-7220. 

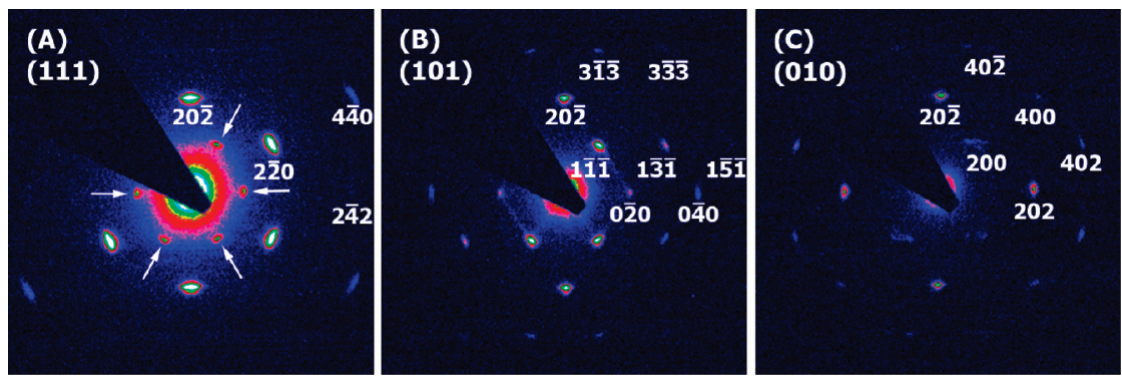

Figure 3. Microradian X-ray diffraction patterns measured with the X-ray beam orthogonal (A) to the substrate $\left(\omega=0^{\circ}\right)$ and after a sample rotation around the vertical axis by $\omega=-35.3^{\circ}(\mathrm{B})$ and $\omega=54.7^{\circ}(\mathrm{C})$. These patterns correspond to the index zones (111), (101), and (010) of the fcc structure. The colloidal crystal is obtained on the cathode at $U=1.5 \mathrm{~V}$. Peaks, which cannot be assigned to the fcc lattice, are marked by arrows in panel A.
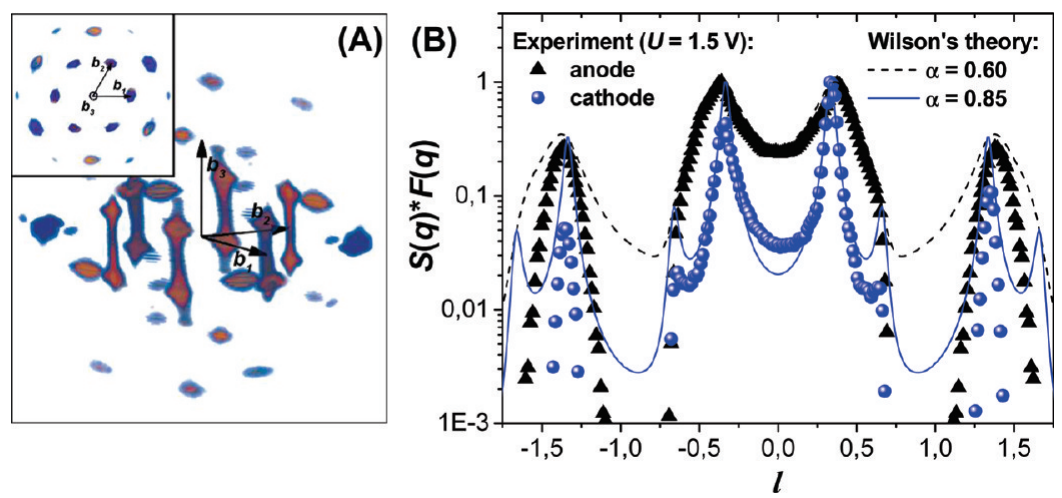

Figure 4. (A) $3 \mathrm{D}$ reconstruction of the reciprocal space for the colloidal crystal obtained on the cathode at $U=1.5 \mathrm{~V}$ (A). The reflections only within $q<0.03 \mathrm{~nm}^{-1}$ are shown. The hexagonal basis is depicted by the vectors $\boldsymbol{b}_{\mathbf{1}}, \boldsymbol{b}_{\mathbf{2}}$, and $\boldsymbol{b}_{\mathbf{3}}$. The inset illustrates a view on the reciprocal space along $\boldsymbol{b}_{3}$. (B) Normalized intensity variation along rods for two samples prepared on electrodes with opposite polarization at $1.5 \mathrm{~V}$. Intensity profiles calculated within Wilson's theory for $\alpha$ equal to 0.85 and 0.60 are shown by solid and dashed lines, respectively.

space is shown in Figure 4A for the colloidal crystal formed on a cathode at $U=1.5 \mathrm{~V}$. One clearly sees the presence of the extended rods of diffuse scattering and of localized reflections with welldefined round shape. This reciprocal lattice is typical for a closepacked structure with stacking faults. ${ }^{37-40}$

We analyze the distribution of the diffracted intensity along the Bragg rod with help of Wilson's theory, ${ }^{37,38}$ which assumes a crystal consisting of a random sequence of close-packed layers. The main parameter of this theory, $\alpha$, is the probability of finding a close-packed layer in the fcc environment. The value of $\alpha$ can be evaluated from the distribution of scattered intensity along a Bragg rod.

Figure 4B shows the normalized intensity profiles along a Bragg rod extracted from 3D reconstruction for colloidal crystals grown at $U=1.5 \mathrm{~V}$ on the cathode and the anode. These profiles are compared to the predictions of Wilson's theory ${ }^{37}$ with different values of the probability $\alpha$ of finding an fcc sequence of stacked layers. The calculated intensity profiles $I(l) \propto S(l) F(l)$, where $S(l)$ is a structure factor and $F(l)$ is the form factor of $5 \%$ polydisperse uniform spheres, are shown by lines in Figure 4B. One can see that while for the crystal grown on the cathode the stacking is mostly fcc, a nearly random hexagonal close packed (rhcp) structure is found on the anode $(\alpha \approx 0.6)$. The dependence of the parameter $\alpha$ on the applied potential is summarized in Figure 5A. Since stacking disorder deteriorates the optical properties of photonic crystals, ${ }^{3,4}$ application of cathode polarization will allow improvement of the crystal quality.

(38) Wilson, A. J. C. Proc. R. Soc. London, Ser. A 1941, 180, 277-285.

(39) Versmold, H. Phys. Rev. Lett. 1995, 75, 763-767.

(40) Petukhov, A. V.; Dolbnya, I. P.; Aarts, D. G.; Vroege, G. J.; Lekkerkerker, H. N. Phys. Rev. Lett. 2003, 90, 028304.
The monotonic improvement of the crystal quality can also be derived from the width of the diffraction spots. The results obtained from the patterns measured at $\omega=0^{\circ}$ are summarized in Figure 5B,C. The full-width at half-maximum (fwhm) of the diffraction maxima in the azimuthal $(\delta \varphi)$ and radial $(\delta q)$ directions characterizes the mosaicity of the colloidal films and the average crystallite size $(\Lambda)$, respectively. One can see that the mosaicity $\delta \varphi$ of the colloidal crystals decreases from $12^{\circ}$ to $5^{\circ}$ when the applied voltage changes from $+1.5 \mathrm{~V}$ to $-1.5 \mathrm{~V}$. Higher values of applied potential lead to significant disorientation of the domains in colloidal films grown at both anode and cathode polarizations. We believe that the given behavior is connected with water electrolysis accompanied by gas evolution on a substrate's surface at high values of $U$. The apparent peak width in the radial direction $\delta q_{\text {app }}$ is determined from the fit by a Lorenzian. The intrinsic peak width $\delta q_{\text {intr }}$ was subsequently estimated assuming the following relation:

$$
\left(\delta q_{\text {app }}\right)^{2}=\Delta^{2}+\left(\delta q_{\text {intr }}\right)^{2}
$$

where $\Delta$ is the instrument resolution $\Delta=(3.9 \pm 0.2) \times 10^{-4} \mathrm{~nm}^{-1}$ (fwhm of the profile of the direct beam). One can see in Figure $5 \mathrm{C}$ that the application of a negative potential as far as $U \geq-1.5 \mathrm{~V}$ leads to a significant increase of the average size of crystallites $\Lambda=$ $2 \pi \mathrm{B} / \delta q_{\text {intr }}$, where $\mathrm{B}$ is a factor of the order 1 . Then gas evolution process at $|U| \geq 2 \mathrm{~V}$ leads to rapid decrease of domain's size, which manifest itself in increase of radial width of diffraction spots.

Thus, the increase of a cathode polarization leads to the improvement of crystal quality, but at the same time the thickness of a film decreases (see Figure 2). In order to prevent gas evolution 


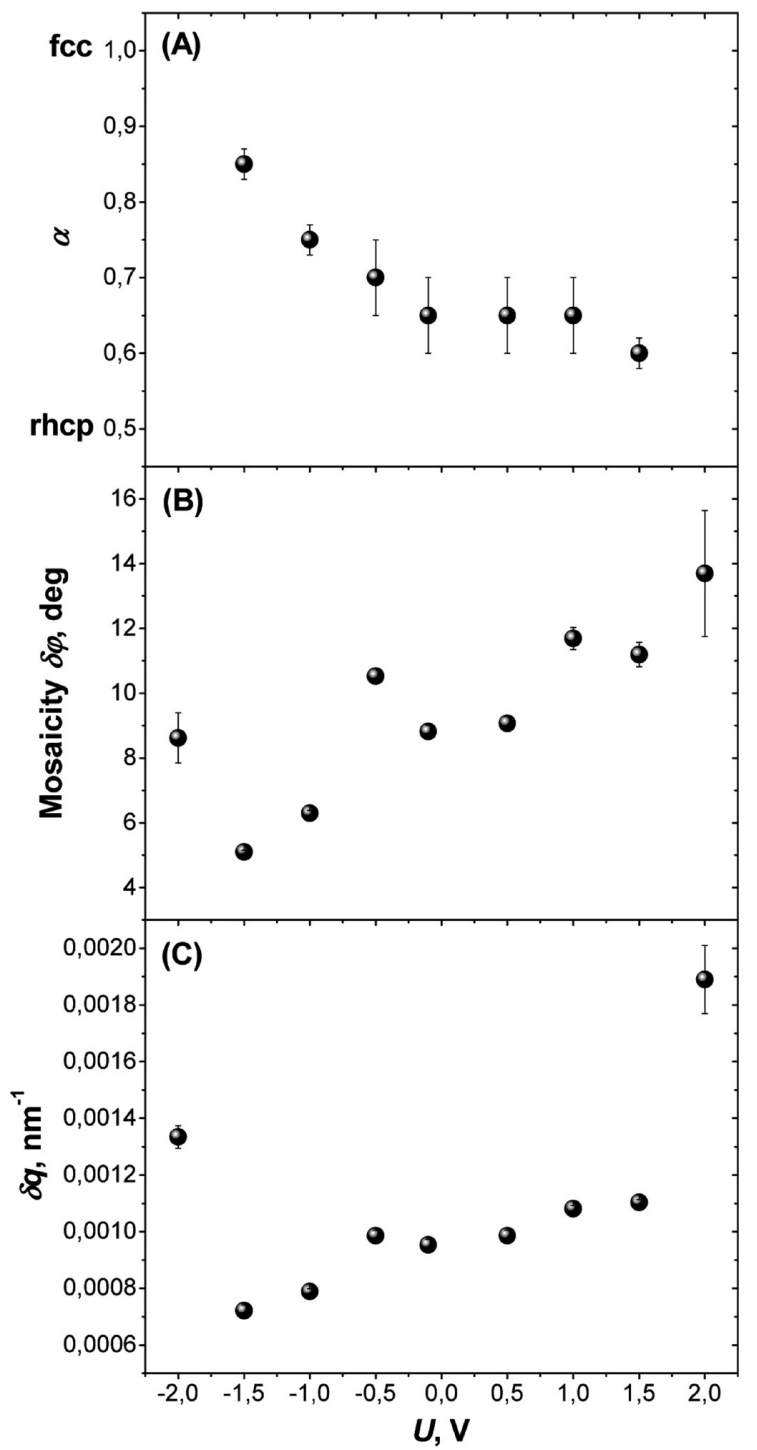

Figure 5. $U$ dependence of the main structural parameters of artificial opals according to microradian X-ray diffraction data: stacking probability $\alpha(\mathrm{A})$, mosaicity of the structure (B), and radial width of (220) reflexes (C). Negative values of $U$ correspond to a cathode polarization, and positive to an anode polarization.

on the electrodes, which is responsible for structural defects, the high values of applied potentials should be avoided. The optimum value of an applied voltage $U$ for the conditions we have used (given concentration of the suspension, $\mathrm{pH}$, charge of colloidal particles, temperature, distance between electrodes, etc.) is around $-1.5 \mathrm{~V}$. These conditions allow growing crystals that are 20 layers thick and have dominant fcc stacking $(\alpha \approx 0.85)$.

The crystal quality of the best sample obtained in the present work (see for example colloidal film grown on cathode at $1.5 \mathrm{~V}$ ) is comparable with the quality of artificial opals grown on glass at optimal conditions, ${ }^{36}$ as for example, the mosaicity is $8.4^{\circ}$ for a crystal grown on glass under optimal conditions, while the same parameter for the best sample obtained by the suggested method on ITO is $5^{\circ}$. The comparison for $\delta q_{\text {intr }} / q_{220}$ values leads to the same conclusion: $\delta q_{\text {intr }} / q_{220}$ equal to 0.068 and 0.025 for films grown on glass and ITO, respectively.

The quality of the obtained colloidal crystals reflects on their optical properties. Figure 6 shows reflectance spectra for the colloidal crystals obtained at $U=1 \mathrm{~V}$ with opposite polarizations. The high reflectance baseline over $1300 \mathrm{~nm}$ is caused by high

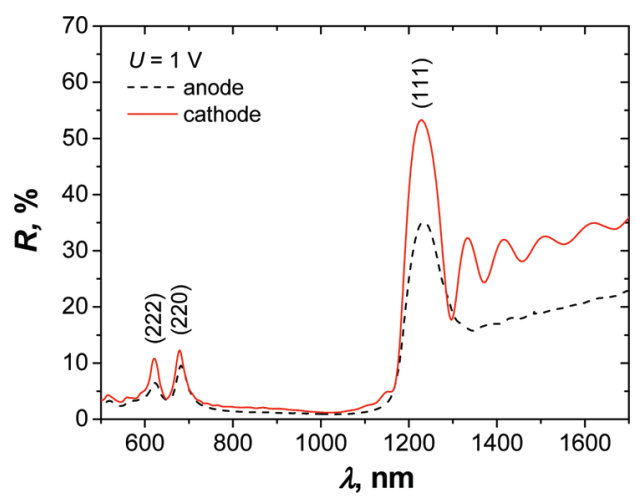

Figure 6. Optical reflection spectra for artificial opals fabricated on ITO. The spectra were measured at incident angle of $8^{\circ}$ with respect to the normal to the sample. A beam with a size of $4 \times$ $4 \mathrm{~mm}^{2}$ was utilized. The samples were obtained at $U=1 \mathrm{~V}$.

reflectivity of light from ITO in this spectral region. The reflection spectra exhibit a main peak at $1230 \mathrm{~nm}$ with two additional low intensity peaks at 680 and $620 \mathrm{~nm}$. These optical stop bands originate to the Bragg reflection caused by the (111), (220), and (222) planes of the fcc structure, respectively. For the most intense reflectance, the reflectivity peak amplitude exceeds $50 \%$ and falls down to $35 \%$ for crystals obtained on the negative and the positive electrode, respectively. Moreover, in the case of the colloidal crystal obtained on the cathode, Fabry-Perot oscillations are observed in the low-energy region, corresponding to a constant thickness of the sample over the beam area $\left(4 \times 4 \mathrm{~mm}^{2}\right)$. The thickness of the colloidal film $(h)$ can be calculated from the position of the Fabry-Perot oscillations as

$$
h=\frac{\lambda_{i}}{2 n_{\text {eff }} \cos \theta\left(1-\frac{\lambda_{i}}{\lambda_{i+1}}\right)}
$$

where $\lambda_{i}$ and $\lambda_{i+1}$ are positions of neighboring reflection maxima $\left(\lambda_{i}<\lambda_{i+1}\right), \theta$ is the incident angle of light with respect to the normal to the sample, and $n_{\text {eff }}$ is the effective (average) refractive index of the polystyrene/air medium. $n_{\mathrm{eff}}$ is defined as

$$
n_{\mathrm{eff}}=\sqrt{n_{\mathrm{PS}}^{2} f_{\mathrm{PS}}+n_{\mathrm{air}}^{2}\left(1-f_{\mathrm{PS}}\right)}
$$

where $f_{\mathrm{PS}} \approx 0.74$ is the volume fraction occupied by polystyrene in a closed packed structure; $n_{\mathrm{PS}} \approx 1.57$ and $n_{\text {air }} \approx 1$ are refractive indexes of polystyrene and air in the near IR region, respectively. ${ }^{41}$ Thus, for colloidal closed packed crystals made of polystyrene microspheres, $n_{\text {eff }}$ is equal to 1.44 .

According to eq 2, the observed peak positions at $\lambda_{1}=1335 \mathrm{~nm}$ and $\lambda_{2}=1415 \mathrm{~nm}$ correspond to $h=8.3 \mu \mathrm{m}$ for the colloidal film obtained on the cathode at $U=1 \mathrm{~V}$. In assumption of an average sphere diameter of $530 \mathrm{~nm}$ (according to the SEM data), the crystal consists of 19 layers. The calculated value is in good agreement with SEM data (see Figure 2).

Finally, it should be noted that the tendencies found for colloidal crystals on ITO substrates have also clearly been seen for other kinds of conducting substrates, for example, glass, mica, and silicon single crystals covered by a thin layer of gold.

\section{Conclusions}

Our observations reveal the effect of an external electric field on the structure of colloidal crystals made by the vertical deposition

(41) Kasarova, S. N.; Sultanova, N. G.; Ivanov, C. D.; Nikolo, I. D. Opt. Mater. 2007, 29, 1481-1490. 
method. Although we do not directly compare the quality of our crystals to those grown on dielectric substrates, our results unambiguously show that application of a negative potential can significantly improve the quality of the synthetic opals. The suggested synthetic approach is a highly reproducible way for the formation of large-scale high-quality dry colloidal crystals with controllable thickness on conducting substrates. This kind of sample is attractive as a template material for preparation of inverse photonic crystals by the electrochemical approach. ${ }^{42,43}$ The applicability of the suggested method for preparation of colloidal crystals on dielectric substrates should be clarified in future experiments.

Characterization of the structure of artificial opals benefits a lot when standard techniques, such as SEM, are complemented by microradian X-ray diffraction. Short acquisition times, modern

(42) Braun, P. V.; Wiltzius, P. Adv. Mater. 2001, 13, 482-485.

(43) Meng, X. D.; Al-Salman, R.; Zhao, J. P.; Borissenko, N.; Li, Y.; Endres, F. Angew. Chem., Int. Ed. 2009, 48, 2703-2707. area detectors, and progress in computing techniques make 3D reconstructions of the reciprocal space routinely available. This method provides extremely valuable information on a real structure of mesoscopic materials, which cannot be easily obtained by other analytical approaches.

Acknowledgment. This work is partially supported by the Russian Federal Agency of Science and Innovations (Grant Nos. 02.740.11.0135 and 02.513.12.3001) and Russian Foundation for Basic Research. We thank the personnel of the DUBBLE beamline and, in particular, Dirk Detollenaere for their excellent support, and Swiss-Norwegian Beamlines for allocation of part of the beam time in the frame of the SNBLDUBBLE agreement. The authors are grateful to G. A. Tsirlina (Moscow State University) for numerous fruitful discussions. The "Nederlandse organisatie voor Wetenschappelijk Onderzoek (NWO)" is thanked for granting us the beam time. 